\title{
Anti-citrullinated protein antibodies and bone loss in patients with early arthritis: comment on the article "Anti-citrullinated protein antibodies and high levels of rheumatoid factor are associated with systemic bone loss in patients with early untreated rheumatoid arthritis" by Bugatti et al.
}

\author{
Santos Castañeda* (D), Irene Llorente, Rosario García-Vicuña and Isidoro González-Álvaro
}

See related Research by Bugatti et al. https://arthritisresearch.biomedcentral.com/articles/10.1186/s13075-016-1116-9

We read with great interest the article about the relationship between anti-citrullinated protein antibodies (ACPA), rheumatoid factor (RF) and systemic bone loss in 155 patients with early untreated rheumatoid arthritis (RA), published recently in Arthritis Research \& Therapy [1]. The work is really interesting since it shows the association of ACPA with low bone mass in both lumbar spine and total hip in patients with early RA [1]. These findings are supported by recent investigations demonstrating the expression of citrullinated antigens on the surface of osteoclastic linage cells, which converts these cells into prime targets for circulating ACPA [2, 3].

In this regard, we want to reinforce these findings with data from the baseline visit of 578 patients from our early arthritis (EA) register, the "Princesa Early Arthritis Register Longitudinal (PEARL)". Briefly, our data show similar results to those described in Bugatti et al.'s work [1], with the difference that we didn't find any association with RF although our population is larger than theirs and includes early RA and undifferentiated arthritis, strengthening the importance of ACPA in the pathogenesis of bone destruction in inflammatory processes [4]. It is important to emphasize that our series is probably very similar

\footnotetext{
* Correspondence: santos.castaneda@salud.madrid.org; scastas@gmail.com Rheumatology Division, Hospital de La Princesa, IIS-IP, Universidad Autónoma, c/Diego de León 62, 28006 Madrid, Spain
}

genetically to Bugatti et al.'s [1], with comparable demographic and intrinsic characteristics of the disease [4].

Furthermore, in our work there were no differences in juxtaarticular bone mineral density (BMD) at the metacarpophalangeal joints between ACPA-positive and ACPAnegative patients, suggesting that active inflammation was not the main mechanism of systemic bone loss in early stages of the disease [4].

Additionally, we also analyzed the levels of anti-mutated citrullinated vimentin IgG antibodies, as they could develop a greater pathogenic role [2]. However, their predictive value for bone loss did not improve the results obtained with ACPA, despite the good correlation between the serum levels of both autoantibodies [4].

At the time we submitted our data for publication, no previous publications had described the association of ACPA and BMD in patients with EA. In addition, considering the scarce current evidence of the effect of ACPA on systemic BMD, we believe that the fact that two independent groups report similar findings in such a close time frame make these results more reliable and challenging.

Recently, Orsolini et al. [5] published similar data in a cohort of patients with established RA, demonstrating a titer-dependent effect of ACPA on systemic bone mass, especially at femoral sites (cortical bone), supporting the above-mentioned findings. 


\section{Authors' response}

\section{Serena Bugatti and Carlomaurizio Montecucco}

We are grateful to Castañeda and colleagues for their interest in our paper recently published in Arthritis Research \& Therapy [1]. Increasing evidence links the presence and the characteristic of autoantibodies to relevant pathophysiological processes in rheumatoid arthritis (RA), also offering a causal explanation of the long-known epidemiological association between seropositivity and worse outcomes [6]. In this direction, the discovery of a direct pro-osteoclastic effect of anti-citrullinated peptide antibodies (ACPA) [2] has revolutionized the assumption that tissue damage in RA depends exclusively on the action of pro-inflammatory cytokines. Following that publication, clinical scientists have put great effort into exploring the early effects of autoantibodies on bone. Individuals with ACPA but no evidence of synovitis show cortical bone changes at the metacarpophalangeal joints [7], and, similarly, we have found that patients with a very short history of RA present reduced systemic bone mineral density only in association with RA-specific autoantibodies [1]. Nicely, in their letter Castañeda and colleagues report similar findings obtained in a large cohort of patients with early arthritis, reinforcing the concept that local and generalized bone remodeling in RA may be at least in part disconnected from classic inflammatory pathways, and that strategies aimed at halting/preventing bone loss should include close monitoring of autoantibodypositive subjects beyond the control of disease activity.

Research on the causal relationship between autoantibodies and RA pathology is in its infancy, and many aspects need further clarification. Among these, the role of rheumatoid factor (RF) remains debated. Whilst we and others have reported a potential additive effect of RF on the background of ACPA-positivity, Castañeda et al. failed to find an association between RF and systemic bone loss. Compared to ACPA, RF testing shows much higher variability in the clinical setting due to greater fluctuations in levels in the same patient over time and to larger differences in test characteristics among different laboratories. These issues, along with the inclusion of non-RA patients in Castañeda et al.'s series, may partially explain the conflicting results. However, the pentameric IgM RF activates complement and enhances, in a dose-dependent way, the pro-inflammatory effect of ACPA on macrophages in vitro, and several clinical observations link high RF levels with more active and more destructive disease [6]. Further research in this field is welcome, as it would translate into better knowledge on the role of autoantibodies as biomarkers and possible therapeutic targets in RA.

\section{Abbreviations}

ACPA: Anti-citrullinated protein antibodies; BMD: Bone mineral density; EA: Early arthritis; PEARL: Princesa Early Arthritis Register Longitudinal; RA: Rheumatoid arthritis; RF: Rheumatoid factor

\section{Acknowledgements}

We would like to thank Teresa Velasco for her invaluable help in the Early Arthritis Clinic and Vanessa Centeno Talayero for technical support.

\section{Funding}

Ministerio de Economía and Competitividad (Instituto de Salud Carlos III, SPAIN) and Fondo Europeo de Desarrollo Regional (FEDER) (grant number: PI12/01578).

\section{Availability of data and materials}

The datasets belonging to our original research are available from the corresponding author on reasonable request.

\section{Authors' contributions}

SC, IL, RG-V and IG-G have equally contributed to the preparation, writing and revision of this Letter. All authors read and approved the final manuscript.

\section{Authors' information}

Not applicable.

\section{Competing interests}

The authors declare that they have no competing interests.

\section{Consent for publication}

Not applicable.

\section{Ethics approval and consent to participate}

The PEARL study is conducted according to the principles expressed in the Helsinki Declaration of 1983 and was approved by the Research Ethics Committee of Hospital Universitario de La Princesa. All patients signed a written consent at study entry.

\section{Publisher's Note}

Springer Nature remains neutral with regard to jurisdictional claims in published maps and institutional affiliations.

Published online: 03 July 2017

\section{References}

1. Bugatti S, Bogliolo L, Vitolo B, Manzo A, Montecucco C, Caporali R. Anti-citrullinated protein antibodies and high levels of rheumatoid factor are associated with systemic bone loss in patients with early untreated rheumatoid arthritis. Arthritis Res Ther. 2016;18:226.

2. Harre U, Georgess D, Bang H, Bozec A, Axmann R, Ossipova E, et al. Induction of osteoclastogenesis and bone loss by human autoantibodies against citrullinated vimentin. J Clin Invest. 2012;122:1791-802.

3. Malmström V, Catrina Al, Klareskog L. The immunopathogenesis of seropositive rheumatoid arthritis: from triggering to targeting. Nat Rev Immunol. 2017;17:60-75.

4. Llorente I, Merino L, Ortiz AM, Escolano E, González-Ortega S, García-Vicuña R, et al. Anti-citrullinated protein antibodies are associated with decreased bone mineral density: baseline data from a register of early arthritis patients. Rheumatol Int. 2017;37:799-806. doi: 10.1007/s00296-017-3674-9 [Epub ahead of print].

5. Orsolini G, Caimmi C, Viapiana O, Idolazzi L, Fracassi E, Gatti D, et al. Titerdependent effect of anti-citrullinated protein antibodies on systemic bone mass in rheumatoid arthritis patients. Calcif Tissue Int. 2017;101:17-23. doi: 10.1007/s00223-017-0253-8 [Epub ahead of print].

6. Bugatti S, Bogliolo L, Montecucco C, Manzo A. B cell autoimmunity and bone damage in rheumatoid arthritis. Reumatismo. 2016;68:117-25.

7. Kleyer A, Finzel S, Rech J, Manger B, Krieter M, Faustini F, et al. Bone loss before the clinical onset of rheumatoid arthritis in subjects with anti citrullinated protein antibodies. Ann Rheum Dis. 2014;73:854-60. 